\title{
Ellipticity of words
}

\author{
Consuelo Martínez* \\ Departamento de Matemáticas, Universidad de Oviedo, \\ C/ Calvo Sotelo, s/n, 33007 Oviedo SPAIN \\ Dedicated to Efim Zelmanov on his 60th birthday
}

\begin{abstract}
We prove that an arbitrary word $v$ of the free pro-p-group is elliptic on a pro- $p$-completion of a discrete finitely generated torsion residually- $p$ group.
\end{abstract}

\section{Introduction}

Let $p$ be a prime number. We say that a group $G$ is residually- $p$ if

$$
\bigcap\left\{H \triangleleft G|| G: H \mid=p^{k}, k \geq 1\right\}=(1) .
$$

In this case the subgroups $H$ of the above intersection can be viewed as a basis of neighborhoods of 1 , thus making $G$ a topological group. If the topology is complete, then we say that $G$ is a pro-p-group. If not, then the completion $G_{\hat{p}}$ of the group $G$ is called the pro-p-completion of $G$.

Let $F(\infty)$ be the free group on the countable set of generators $x_{1}, x_{2}, \ldots$. The pro- $p$-completion $F=F(\infty)_{\hat{p}}$ is called a free pro- $p$-group on $x_{1}, x_{2}, \ldots$, We call an element $w \in F$ a word if it involves finitely many generators.

*Partially supported by MTM 2013-45588-C3-1-P and GRUPIN 14-142 
Let $G$ be a pro- $p$-group and let $w=w\left(x_{1}, \ldots, x_{n}\right) \in F$. Consider

$$
w(G)=\left\{w\left(g_{1}, \ldots, g_{n}\right) \mid g_{i} \in G, 1 \leq i \leq n\right\}
$$

the set of values of the word $w$ in $G$ and let $\langle\overline{w(G)}\rangle$ denote the closed subgroup of $G$ generated by the set $w(G)$.

We say (see $[\mathrm{S}]$ ) that the word $w$ is elliptic (or has finite verbal width) on $G$ if there exists $N \geq 1$ such that $\langle\overline{w(G)}\rangle=\underbrace{w(G)^{ \pm 1} \cdots w(G)^{ \pm 1}}_{N}$.

This is equivalent to say that the discrete subgroup of $G$ generated by $w(G)$ is closed in $G$.

It is known that on some important classes of pro-p-groups ( $p$-adic analytic pro-p-groups [JZ], the Nottingham group [Kl]) all words are elliptic.

In the first chapter we prove the following theorem

Theorem 1.1 Let $\Gamma$ be a finitely generated residually-p torsion group. Then an arbitrary word $w \in F$ is elliptic on $G=\Gamma_{\hat{p}}$.

We remark that the celebrated groups of Golod-Shafarevich, Grigorchuk or Gupta-Sidki are residually- $p$ torsion groups.

In the second chapter we prove a stronger statement for the so called multilinear words.

Let $w=w\left(x_{1}, \ldots, x_{n}\right) \in F$. Let $G$ be a pro-p-group. Choose elements $a_{1}, \ldots, a_{i-1}, a_{i+1}, \ldots, a_{n} \in G$ and fix all variables $x_{j}=a_{j}$ except one $x_{i}$. Denote $\alpha=\left(a_{1}, \ldots, a_{i-1}, a_{i+1}, \ldots, a_{n}\right)$ and consider the set $w(G, i, \alpha)=$ $\left\{w\left(a_{1}, \ldots, a_{i-1}, g, a_{i+1}, \ldots, a_{n}\right) \mid g \in G\right\}$.

Definition 1.1 We say that the element $w$ is strongly elliptic on $G$ if there exist finite subsets $M_{i} \subseteq \underbrace{G \times \cdots \times G}_{n-1}, 1 \leq i \leq n$, and an order on $\cup_{i=1}^{n} M_{i}=\left\{\alpha_{1}<\alpha_{2}<\cdots<\alpha_{q}\right\}, \alpha_{k} \in M_{i_{k}}, 1 \leq k \leq q, 1 \leq i_{k} \leq n$, such that the verbal subgroup $\langle\overline{w(G)}\rangle$ is equal to

$$
w\left(G, i_{1}, \alpha_{1}\right)^{ \pm 1} \cdots w\left(G, i_{q}, \alpha_{q}\right)^{ \pm 1} .
$$


Clearly, if a word is strongly elliptic on $G$ then it is elliptic.

By the result of J. P. Serre [Se] the commutator is strongly elliptic on finitely generated pro- $p$-groups.

Consider the lower central series of $F, F=F_{1} \geq F_{2} \geq \cdots$, and the Zassenhaus series $F=F_{(1)} \geq F_{(2)} \geq \cdots \geq F_{(n)} \geq \cdots$, where $F_{(n)}$ is the subgroup of $F$ generated by all powers $g^{p^{j}}, g \in F_{i}, i p^{j} \geq n$. Both series (central series) lead to Lie algebras $L(F)=\bigoplus_{i \geq 1} F_{i} / F_{i+1}$ and $L_{p}(F)=\bigoplus F_{(i)} / F_{(i+1)}$.

Definition 1.2 Let $w \in F$ be a word. Suppose that $w \in F_{n} \backslash F_{n+1}$. We call the word $w$ multilinear if $w=\bar{w} w^{\prime}$, where

(i) $\bar{w}$ is a product of powers of left-normed commutators,

$$
\bar{w}=\prod_{\sigma \in S_{n}}\left[\cdots\left[x_{\sigma(1)}, x_{\sigma(2)}\right], \cdots, x_{\sigma(n)}\right]^{k_{\sigma}},
$$

where $k_{\sigma} \in \mathbb{Z}_{p}$ are $p$-adic integers such that the element $\bar{w} F_{n+1}$ does not belong to $p L(F)$,

(ii) the element $w^{\prime} \in F_{n+1}$ is a (converging) product of commutators in $x_{1}, \ldots, x_{n}$ of length $\geq n+1$, each commutator involves all $n$ generators $x_{1}, \ldots, x_{n}$.

Theorem 1.2 Let $\Gamma$ be a finitely generated residually-p torsion group. Then an arbitrary multilinear word is strongly elliptic on the pro-p-group $G=\Gamma_{\hat{p}}$, the pro-p-completion of $\Gamma$.

A linearization process leads to

Theorem 1.3 For an arbitrary nonidentical word $w \in F$ there exist a multilinear element $\tilde{w} \in\langle\overline{w(F)}\rangle$.

\section{$2 \quad$ Verbally just infinite pro- $p$-groups}

We say that a pro-p-group $G$ is verbally just infinite if $G$ is infinite and for an arbitrary word $w \in F$ either $w(G)=1$ or $|G:\langle\overline{w(G)}\rangle|<\infty$.

Lemma 2.1 Let $G$ be a finitely generated verbally just infinite pro-pgroup. Then an arbitrary word $w \in F$ is elliptic on $G$ or $G$ is virtually abelian. 
Proof. Let $G$ be a finitely generated pro-p-group that is not virtually abelian. Let $w \in F, H=\langle\overline{w(G)}\rangle$. If $H=(1)$, then the word $w$ is clearly elliptic on $G$. If $H \neq(1)$ then the subgroup $H$ has finite index in $G$ and, therefore, is a finitely generated pro- $p$-group.

Since the group $G$ is not virtually abelian it follows that $[H, H] \neq(1)$. By the result of J. P. Serre [Se], the subgroup $[H, H]$ is closed in $G$.

Consider the word

$$
v\left(x_{1}, \ldots, x_{m}, y_{1}, \ldots, y_{m}\right)=\left[w\left(x_{1}, \ldots, x_{m}\right), w\left(y_{1}, \ldots, y_{m}\right)\right] .
$$

Clearly, $[H, H]=\langle\overline{v(G)}\rangle$. By the assumption of the lemma, $|G:[H, H]|<\infty$.

Let $h_{1}, \ldots, h_{r} \in w(G)^{ \pm 1}$ be a maximal system of elements that are distinct module $[H, H]$. Then $h_{1}, \ldots, h_{r}$ generate $H$. By the result of J. P. Serre [Se] we have

$$
[H, H]=\left[H, h_{1}\right] \cdots\left[H, h_{r}\right] .
$$

Every element of $H$ can be represented as $h_{i_{1}} \cdots h_{i_{t}}, t \leq|H:[H, H]|$, modulo $[H, H]$. Hence

$$
H=\bigcup h_{i_{1}} \cdots h_{i_{t}}\left[H, h_{1}\right] \cdots\left[H, h_{r}\right]
$$

Now the verbal width of $w$ is $\leq|H:[H, H]|+2 r$ since $\left[x, h_{i}\right]=\left(h_{i}^{x}\right)^{-1} h_{i} \in$ $w(G)^{ \pm 1} \cdot w(G)^{ \pm 1}$. This completes the proof of the lemma.

Remark. A finitely generated virtually abelian pro-p-group $G$ is $p$-adic analytic. If $w \in F(\infty)$ then $w$ is elliptic on $G$ by the result of A. JaikinZapirain [JZ]. Most likely this is also the case when $w \in F \backslash F(\infty)$, but there are no references.

Let $G$ be a pro-p-group and let $1 \neq w \in F$. We say that the group $G$ satisfies the pro- $p$ - identity $w=1$ if $w(G)=(1)$.

The following theorem was proved by E. Zelmanov ( $[\mathrm{Z} 3])$.

Theorem 2.1 Let $G$ be a pro-p-group satisfying a nontrivial pro-p-identity and having a dense finitely generated torsion discrete subgroup. Then $|G|<$ $\infty$. 
Lemma 2.2 Let $\Gamma$ be a finitely generated residually-p torsion group, $G=$ $\Gamma_{\hat{p}}$. Then either $|\Gamma|<\infty$ or $G$ is a verbally just infinite group.

Proof. Suppose that the groups $\Gamma$ and $G$ are infinite. Let $1 \neq w=$ $w\left(x_{1}, \ldots, x_{n}\right) \in F$ be a word. We need to show that the subgroup $H=$ $\langle\overline{w(G)}\rangle$ has finite index in $G$.

Consider the pro- $p$-group $G^{\sharp}=G / H$ and the discret subgroup $\Gamma^{\sharp}=$ $\Gamma H / H$. The group $\Gamma^{\sharp}$ is finitely generated, torsion and dense in $G^{\sharp}$. The pro-p-group $G^{\sharp}$ satisfies a non trivial pro-p-identity. Then, by Zelmanov's theorem , $\left|G^{\sharp}\right|<\infty$, what finishes the proof of the lemma.

\section{Proof of Theorem 1.1}

Let $\Gamma$ be a finitely generated residually- $p$ torsion group and let $G=\Gamma_{\hat{p}}$ be its pro-p-completion. Let $w \in F$ be a word. If $|\Gamma|<\infty$ then the result is obvious. Suppose therefore that $\Gamma$ is infinite. By Lemma 2.2 the pro- $p$ group $G$ is verbally just infinite. We remark that the group $G$ is not virtually abelian since then a finitely generated torsion virtually abelian group $\Gamma$ would be finite. Now Lemma 2.1 implies the word $w$ is elliptic on $G$.

\section{Multilinear words}

Let's recall the construction of a Lie ring associated to a pro-p-group $G$.

Consider the lower central series $G=G_{1}>G_{2}>\cdots$ of the group $G$, $G_{n+1}=\left[G_{n}, G\right]$. Clearly $\left[G_{i}, G_{j}\right] \subseteq G_{i+j}$, the abelian group $G_{i} / G_{i+1}$ is a module over the ring of p-adic integers $\mathbb{Z}_{p}$.

Consider the direct sum

$$
L(G)=\oplus_{i \geq 1} G_{i} / G_{i+1}
$$

The Lie ring multiplication is defined on homogeneous elements by

$$
\left[a G_{i+1}, b G_{j+1}\right]=[a, b] G_{i+j+1}
$$

and extended to arbitrary elements by linearity. This makes $L(G)$ a Lie algebra over $\mathbb{Z}_{p}$. 
For the free pro-p-group $F$ the Lie algebra $L(F)$ is known to be the free Lie $\mathbb{Z}_{p}$-algebra on the set of free generators $\bar{X}=\left\{\bar{x}_{i}=x_{i} F_{2} \mid i \geq 1\right\}$

Along with the lower central series of $F, F=F_{1} \geq F_{2} \geq \cdots$, we consider the Zassenhaus series $F=F_{(1)} \geq F_{(2)} \geq \cdots \geq F_{(n)} \geq \cdots$, where $F_{(n)}$ is the subgroup of $F$ generated by all powers $g^{p^{j}}, g \in F_{i}, i p^{j} \geq n,\left[F_{(i)}, F_{(j)}\right] \subseteq$ $F_{(i+j)}, \cap_{i \geq 1} F_{(i)}=(1)$, each factor $F_{(i)} / F_{(i+1)}$ is an elementary abelian p-group. The Zassenhaus series gives rise to the Lie algebra $L_{p}(F)=\bigoplus F_{(i)} / F_{(i+1)}$ over the field $\mathbb{Z} / p \mathbb{Z}$.

\section{Proof of Theorem 1.3}

The proof of this result imitates the well known linearization process in algebras (see [SSSZ]) or, equivalently, Higman's collection process in groups $[\mathrm{H}]$.

As always we consider the free group $F(\infty)$ and the free pro-p-group $F$ on the set $X=\left\{x_{i} \mid i \geq 1\right\}$ of free generators.

Let $w \in F_{(n)} \backslash F_{(n+1)}$. Replacing $w$ by a group commutator $\left[w, x_{i}\right]$, if necessary, we may assume that $n$ is coprime to $p$. The element $w$ can be represented as $w=\bar{w} w^{\prime}$, where $\bar{w}$ is a product of $\rho^{p^{j}}, \rho$ is a commutator in $X$ of length $i, i p^{j}=n, w^{\prime} \in F_{(n+1)}$.

Since $n$ is coprime to $p$ it follows that all $j=0$, that is, $\bar{w}$ is a product of commutators of length $n$ in $X$. If $\bar{w} F_{n+1} \in p\left(F_{n} / F_{n+1}\right)$ then $\bar{w} \in F_{(n+1)}$, a contradiction.

Let Lie $\langle X\rangle$ denote the free Lie $\mathbb{Z}_{p}$-algebra on the same set of free generators $X$ and $\bar{X}=\left\{\bar{x}_{1}, \bar{x}_{2}, \ldots\right\}$, where $\bar{x}_{i}=x_{i} F_{2}$. Consider the element $f\left(\bar{x}_{1}, \ldots, \bar{x}_{m}\right)=\bar{w} F_{n+1}$ of the free Lie $\mathbb{Z}_{p}$-algebra Lie $\langle\bar{X}\rangle$. The element $f\left(\bar{x}_{1}, \ldots, \bar{x}_{m}\right)$ is homogeneous of total degree $n$ and $f \notin p \operatorname{Lie}\langle\bar{X}\rangle_{n}$. The element $f$ does not, though, have to be homogeneous in each variable $\bar{x}_{i}$.

For a multiindex $d=\left(d_{1}, \ldots, d_{m}\right), d_{i} \geq 0, \sum_{i=1}^{m} d_{i}=n$ let $f_{d}$ be the homogeneous component of $f$ having degree $d_{i}$ in $\bar{x}_{i}, 1 \leq i \leq m$.

For a fixed index $i, 1 \leq i \leq m$, let $f_{i}^{\prime}=\sum_{d_{i} \neq 0} f_{d}, f_{i}^{\prime \prime}=\sum_{d_{i}=0} f_{d}$. Then $f=f_{i}^{\prime}+f_{i}^{\prime \prime}$.

If $f_{i}^{\prime \prime} \notin p \operatorname{Lie}\langle\bar{X}\rangle_{n}$, then considering $w\left(x_{1}, \ldots, x_{i-1}, 1, x_{i+1}, \ldots, x_{m}\right)$ (that lies in $\langle w(F)\rangle)$ instead of $w$ we cut the number of variables. 
If $f_{i}^{\prime} \notin p \operatorname{Lie}\langle\bar{X}\rangle_{n}$, then considering $w:=w w\left(x_{1}, \ldots, x_{i-1}, 1, x_{i+1}, \ldots, x_{m}\right)^{-1}$ instead of $w$ we can assume that $f=\sum_{d_{i} \neq 0} f_{d}$.

Arguing in this way with all generators $x_{1}, \ldots, x_{m}$ we will assume that $f\left(\bar{x}_{1}, \ldots \bar{x}_{m}\right)=\sum f_{d}\left(\bar{x}_{1}, \ldots \bar{x}_{m}\right)$, where for each multiindex $d$ on the right hand side we have $d_{1} \geq 1, \ldots, d_{m} \geq 1$.

Fix a multiindex $d=\left(d_{1}, \ldots, d_{m}\right), d_{i} \geq 1$ such that $f_{d} \notin p \operatorname{Lie}\langle\bar{X}\rangle_{n}$. For each $i, 1 \leq i \leq r$, choose a set of $d_{i}$ elements $X_{i}=\left\{x_{i 1}, \ldots, x_{i d_{i}}\right\} \subset X$ such that the subsets $X_{1}, \ldots, X_{m}$ do not intersect. (If $d_{i}=1$ then we choose $\left.X_{i}=\left\{x_{i}\right\}\right)$.

For a nonempty subset $S \subseteq\left[1, d_{i}\right]$ let $\bar{X}_{i}(S)=\sum_{j \in S} \bar{x}_{i j}$.

For a nonnegative integer $a$, let us define $\operatorname{sgn}(a)=(-1)^{a}$. The element

$$
\begin{gathered}
\tilde{f}=\sum_{\emptyset \neq S_{i} \subseteq\left[1, d_{i}\right]} \operatorname{sgn}\left(\sum_{i=1}^{r}\left(d_{i}-\left|S_{i}\right|\right)\right) f\left(\bar{X}_{1}\left(S_{1}\right), \ldots, \bar{X}_{m}\left(S_{m}\right)\right)= \\
\sum_{\emptyset \neq S_{i} \subseteq\left[1, d_{i}\right]} \operatorname{sgn}\left(\sum_{i=1}^{r}\left(d_{i}-\left|S_{i}\right|\right)\right) f_{d}\left(\bar{X}_{1}\left(S_{1}\right), \ldots, \bar{X}_{m}\left(S_{m}\right)\right)
\end{gathered}
$$

is multilinear in all variables $\bar{x}_{i j}, 1 \leq i \leq m, 1 \leq j \leq d_{i}$.

We call $\tilde{f}$ the complete linearization of $f$, that corresponds to the multiindex $d$. Of course, it depends on the choice of $d$.

We use two facts about complete linearizations over a field (see [SSSZ]):

(1) If $h\left(x_{1}, \ldots, x_{m}\right)$ is a homogeneous (in all variables) element of degree multiindex $d^{\prime}=\left(d_{1}^{\prime}, \ldots, d_{m}^{\prime}\right), d_{i}^{\prime} \geq 1, \sum_{i=1}^{m} d_{i}^{\prime}=\sum_{i=1}^{m} d_{i}$ and $d^{\prime} \neq d$, then the complete linearization corresponding to the multiindex $d$ turns $h$ into 0 ,

(2) let $h$ be a nonzero element of a free Lie (associative) algebra over a field which is homogeneous in all variables. Then the complete linearization of $h$ is $\neq 0$.

The assertions (1), (2) imply that $\tilde{f} \neq 0 \bmod p$.

Now let us imitate the above process in the free pro-p-group. For a nonempty subset $S \subseteq\left[1, d_{i}\right]$ let $X_{i}(S)$ be a product of generators $x_{i j}, j \in S$, in an arbitrary order.

Let $v$ be a product of elements of the form

$$
w\left(X_{1}\left(S_{1}\right), \ldots, X_{m}\left(S_{m}\right)\right)^{\operatorname{sgn}\left(\sum_{i=1}^{m}\left(d_{i}-\left|S_{i}\right|\right)\right)}
$$


in an arbitrary order, where $S_{i}$ run over all nonempty subsets of $\left[1, d_{i}\right]$.

It is straightforward that $v \in F_{(n)}$ and $v F_{(n+1)}=\tilde{f}\left(x_{i j} F_{(2)}\right) \neq 0$.

Changing names of free generators, if needed, we can say that we found a nonidentical element $v \in\langle w(F)\rangle$ such that $v=\bar{v} v^{\prime}$, the element $\bar{v}$ is a product of multilinear commutators $\left[\ldots\left[x_{\sigma(1)}, x_{\sigma(2)}\right], \ldots, x_{\sigma(n)}\right], \sigma \in S_{n}, \bar{v} \notin F_{(n+1)}$, $v^{\prime} \in F_{(n+1)}$.

Collecting at the right end all commutators not involving $x_{1}$, then collecting again, among all those commutators that include $x_{1}$, those that do not involve $x_{2}$ (again at the right end) and so on, finally we can represent $v^{\prime}$ as $v^{\prime}=v^{\prime \prime} v_{n} \cdots v_{2} v_{1}$, where $v^{\prime \prime}, v_{i} \in F_{(n+1)}$, the element $v_{i}$ is a product of commutators (of length $\geq n+1$ ) that involve $x_{1}, \ldots, x_{i-1}$ and do not involve $x_{i}$. The element $v^{\prime \prime}$ is a product of commutators that involve $x_{1}, x_{2}, \ldots, x_{n}$.

Let us show, by induction on $i$, that $v_{i} \in\langle v(F)\rangle$. Substituting $x_{1}=1$ we get $\left.v\right|_{x_{1}=1}=v_{1}$. Hence $v_{1} \in\langle v(F)\rangle$. Now substituting $x_{i}=1$ we get $\left.v\right|_{x_{i}=1}=v_{i}\left(\left.v_{i-1}\right|_{x_{i}=1}\right) \cdots\left(\left.v_{1}\right|_{x_{i}=1}\right)$. Hence $v_{i} \in\langle v(F)\rangle$.

It implies that $\bar{v} v^{\prime \prime} \in\langle v(F)\rangle, v^{\prime \prime}$ is product of commutators of length $\geq n+1$, involving $x_{1}, \ldots, x_{n}$ and of $p$-powers $\left[\ldots\left[x_{\sigma(1)}, x_{\sigma(2)}\right], \ldots, x_{\sigma(n)}\right]^{p^{k}}$, $k \geq 1, \sigma \in S_{n}$. Moving all these p-powers to the left we get

$$
\bar{v} v^{\prime \prime}=\left(\prod_{\sigma \in S_{n}}\left[\ldots\left[x_{\sigma(1)}, x_{\sigma(2)}\right], \ldots, x_{\sigma(n)}\right]^{k_{\sigma}}\right) v^{\prime \prime \prime}, k_{\sigma} \in \mathbb{Z}_{p},
$$

and $v^{\prime \prime \prime}$ is a (converging) product of commutators of length $\geq n+1$ involving all $x_{1}, \ldots, x_{n}$. Substituting 1 for all generators that do not belong to $\left\{x_{1}, \ldots, x_{n}\right\}$ we can assume that all factor commutators in $v^{\prime \prime \prime}$ involve only $x_{1}, \ldots, x_{n}$. This finishes the proof of Theorem 1.3.

In order to prove Theorem 1.2 we will have to adapt the above definitions to Lie algebras.

As above, let $X=\left\{x_{i} \mid i \geq 1\right\}$ and let $\operatorname{Lie}\langle X\rangle$ denote the free Lie $\mathbb{Z}_{p^{-}}$ algebra on the set of free generators $X$. Let $f\left(x_{1}, \ldots, x_{n}\right)$ be a multilinear element from $\operatorname{Lie}\langle X\rangle$ and let $L$ be a Lie algebra over $\mathbb{Z}_{p}$.

Consider the set $f(L)=\left\{f\left(a_{1}, \ldots, a_{n}\right) \mid a_{i} \in L\right\}$ of values of the element $f$ on $L$. 
Definition 3.1 We say that the element $f$ is elliptic on $L$ if there exists $N \geq 1$ such that the $\mathbb{Z}_{p}$-linear span of $f(L)$, Span $f(L)$, is

$$
\operatorname{Span}(f(L))=\underbrace{f(L)+\cdots+f(L)}_{N} .
$$

Definition 3.2 We say that the element $f$ is strongly elliptic on $L$ if there exist finite sets $M_{i} \subseteq \underbrace{L \times \cdots \times L}_{n-1}, 1 \leq i \leq n$, such that $\operatorname{Spanf}(L)$ is a sum of additive subgroups $f\left(a_{1}, \ldots, a_{i-1}, L, a_{i+1}, \ldots, a_{n}\right)$, where $\left(a_{1}, \ldots, a_{i-1}\right.$, $\left.a_{i+1}, \ldots, a_{n}\right) \in M_{i}, 1 \leq i \leq n$.

Clearly a strongly elliptic multilinear element is elliptic.

An element $a$ of a Lie algebra is said to be ad-nilpotent if the linear transformation $\operatorname{ad}(a)$ is nilpotent.

Lemma 3.1 Let $L$ be a Lie $\mathbb{Z} / p^{k} \mathbb{Z}$-algebra generated by a finite subset $X \subseteq L$ such that an arbitrary commutator in $X$ is ad-nilpotent (we consider elements of $X$ as commutators of length 1$)$. Let $f\left(x_{1}, \ldots, x_{n}\right) \in \operatorname{Lie}\langle X\rangle$ be a multilinear element of the free Lie $\mathbb{Z}_{p^{-}}$algebra such that $f \notin p L i e\langle X\rangle$. Then

(i) $I=\operatorname{Spanf}(L)$ is a Lie ideal in $L,|L: \operatorname{Spanf}(L)|<\infty$;

(ii) $f$ is strongly elliptic on $L$

Proof: To see that $I=\operatorname{Spanf}(L)$ is an ideal in $L$ choose arbitrary elements $b, b_{1}, \ldots, b_{n} \in L$. Then

$$
\left[b, f\left(b_{1}, \ldots, b_{n}\right)\right]=f\left(\left[b, b_{1}\right], b_{2}, \ldots b_{n}\right)+\ldots+f\left(b_{1}, b_{2}, \ldots,\left[b, b_{n}\right]\right),
$$

which implies the claim.

The Lie algebra $L / I+p L(1)$ is generated by a finite set ( = image of $X$ ) and every commutator in these generators is ad-nilpotent and (2) satisfies a nontrivial polynomial identity.

By [ Z3] the $\mathbb{Z} / p \mathbb{Z}$-algebra $L / I+p L$ is nilpotent. Hence there exists $s \geq 1$ such that $L^{s} \subseteq I+p L$.

Since $p^{k} L=(0)$ this implies $\left(L^{s}\right)^{k} \subseteq I$.

In $[K, P]$ it is shown that in a finitely generated solvable Lie algebra $L$ if all commutators in generators are ad-nilpotent, then the algebra is nilpotent. 
Hence there exists $d \geq 1$ such that $L^{d} \subseteq\left(L^{s}\right)^{k} \subseteq I$. It implies that $|L: I|<$ $\infty$, which finishes the proof of (i).

Again in $[\mathrm{K}, \mathrm{P}]$ it is shown that in a finitely generated Lie algebra $L$ if all commutators in generators are ad-nilpotent, then each power of $L$ is a finitely generated subalgebra (this result is even valid in rings). Since the algebra $L^{d}$ is finitely generated and $\left|I: L^{d}\right|<\infty$, it follows that the algebra $I$ is finitely generated.

Suppose that $I$ is generated as a Lie algebra by a finite collection of elements $f\left(a_{i_{1}}, \ldots, a_{i_{n}}\right), 1 \leq i \leq r, a_{i j}$ are commutators in generators. We have

$$
\begin{gathered}
I \subseteq \sum_{i}\left(\mathbb{Z} / p^{k} \mathbb{Z}\right) f\left(a_{i_{1}}, \ldots, a_{i_{n}}\right)+\sum_{i}\left[L, f\left(a_{i_{1}}, \ldots, a_{i_{n}}\right)\right] \subseteq \\
\sum f\left(a_{i_{1}}, \ldots, a_{i_{t-1}}, L, a_{i_{t+1}}, \ldots, a_{i_{n}}\right)
\end{gathered}
$$

which means that the element $f$ is strongly elliptic on $L$. This finishes the proof of the lemma.

Let $w=\bar{w} w^{\prime}$ be a multilinear word from $F$,

$$
\bar{w}=\prod_{\sigma \in S_{n}}\left[\cdots\left[x_{\sigma(1)}, x_{\sigma(2)}\right], \cdots x_{\sigma(n)}\right]^{k_{\sigma}}, k_{\sigma} \in \mathbb{Z}_{p}, w^{\prime} \in F_{n+1} .
$$

As we have mentioned earlier, the Lie ring $L(F)=\oplus_{i \geq 1} F_{i} / F_{i+1}$ is a free Lie $\mathbb{Z}_{p}$ algebra on the free generators $\bar{x}_{i}=x_{i} F_{2}, i \geq 1$.

Let $f\left(\bar{x}_{1}, \ldots, \bar{x}_{n}\right)=\sum k_{\sigma}\left[\bar{x}_{\sigma(1)}, \ldots, \bar{x}_{\sigma(n)}\right]=w F_{n+1}$ be a multilinear element from $L(F)=\operatorname{Lie}\langle\bar{X}\rangle$ that corresponds to $w$.

Let $G$ be a pro- $p$-group, let $G=G_{1}>G_{2}>\cdots$ be its lower central series. Let $L=L(G)=\oplus_{i \geq 1} G_{i} / G_{i+1}$ be the Lie algebra linked to the lower central series. As above, $\operatorname{Spanf}(L)$ denotes the $\mathbb{Z}_{p}$-linear span of the set $f(L)$. Since $f$ is multilinear it follows (as in Lemma 3.1) that $\operatorname{Spanf}(L)$ is an ideal of $L$.

Lemma 3.2 If $|L: \operatorname{Span} f(L)|<\infty$ and $f$ is strongly elliptic on $L$, then $|G:\langle\overline{w(G)}\rangle|<\infty$ and $w$ is strongly elliptic on $G$.

Proof. Since $|L: \operatorname{Spanf}(L)|<\infty$, it follows that there is $k \geq 1$ such that $p^{k} L \subseteq \operatorname{Spanf}(L)$. Hence $L / \operatorname{Spanf}(L)$ becomes a $\mathbb{Z} / p^{k} \mathbb{Z}$-module. 
Since the element $f$ is strongly elliptic, there exist finite sets $\bar{M}_{i} \subseteq$ $\underbrace{L \times \cdots \times L}_{n-1}, 1 \leq i \leq n, \bar{M}_{i}=\left\{\left(a_{j, 1}^{(i)}, \ldots, a_{j, i-1}^{(i)}, a_{j, i+1}^{(i)}, \ldots, a_{j, n}^{(i)}\right) \mid 1 \leq j \leq\right.$ $\left.m_{i}=\left|\bar{M}_{i}\right|\right\}$ such that

$$
\operatorname{Spanf}(L)=\sum f\left(a_{j, 1}^{(i)}, \ldots, a_{j, i-1}^{(i)}, L, a_{j, i+1}^{(i)}, \ldots, a_{j, n}^{(i)}\right) .
$$

Without loss of generality we will assume all elements $a_{j, k}^{(i)}$ to be homogenoeus, $a_{j, k}^{(i)} \in L_{d(i, j, k)}$.

Choose elements $g_{j k}^{(i)} \in G_{d(i, j, k)}$ such that $a_{j, k}^{(i)}=g_{j k}^{(i)} G_{d(i, j, k)+1}$.

Let $M_{i}=\left\{\left(g_{j 1}^{(i)}, \ldots, g_{j n}^{(i)}\right), 1 \leq j \leq m_{i}\right\}, 1 \leq i \leq n$.

Choose an arbitrary order in $\bigcup_{i=1}^{n} M_{i}$.

Let $S$ denote the ordered product $S=\prod_{u} w(G, i, u), u \in \bigcup_{i=1}^{n} M_{i}$. For an $(n-1)$-tuple, $u=\left(g_{j 1}^{(i)}, \ldots, g_{j n}^{(i)}\right) \in M_{i}$, denote $d(u)=\sum_{i \neq k=1}^{n} d(i, j, k)$.

Let $g \in G_{r}$. Suppose that the element $g G_{r+1} \in L_{r}$ lies in $\operatorname{Spanf}(L)$. Then $g G_{r+1}=\sum f\left(a_{j, 1}^{(i)}, \ldots, a_{j, i-1}^{(i)}, b_{j i}^{(i)}, a_{j, i+1}^{(i)}, \ldots, a_{j, n}^{(i)}\right)$, where $b_{j i}^{(i)}$ are homogeneous elements of degrees $r-\sum_{i \neq k=1}^{n} d(i, j, k)$.

Choose elements $g_{u} \in G_{r-d(u)}, u=\left(g_{j 1}^{(i)}, \ldots, g_{j, n}^{(i)}\right)$ such that $g_{u} G_{r-d(u)+1}=$ $b_{j i}^{(i)}$. Then $g=\prod_{u} w\left(g_{u}, i, u\right)$ modulo $G_{r+1}, u \in \bigcup_{i=1}^{n} M_{i}$.

From $|L: \operatorname{Spanf}(L)|<\infty$ it follows that there exist $t \geq 1$ and $k \geq 1$ such that $p^{k} L+\sum_{i \geq t} L_{i} \subseteq \operatorname{Spanf}(L)$.

We have $p^{k} L_{1} \subseteq \operatorname{Spanf}(L) \subseteq L_{2}+L_{3}+\cdots$. Hence $p^{k} L_{1}=(0)$.

Let us show that $G_{t} \subseteq S$. Since the set $S$ is closed (because it is the continous image of a compact set) it is sufficient to show that for any $r \geq t$ we have that $G_{t} \subseteq S G_{r}$ (what would imply that $G_{t} \subseteq \bar{S}=S$.)

Let us use induction on $r$. It is clear if $r=t$ ( since $G_{t} \subseteq S G_{t}$ ). Choose $g \in G_{t}$ and suppose that there exists $s \in S$ such that $g s^{-1} \in G_{r}$. Then the element $g s^{-1} G_{r+1}$ lies in the $\operatorname{Spanf}(L)$. Hence, there exist elements $g_{u} \in G_{r-d(u)}$ such that $g s^{-1}=\prod_{u} w\left(g_{u}, i, u\right) \bmod G_{r+1}$.

Let $s=\prod_{u} w\left(g_{u}^{\prime}, i, u\right)$. Then

$$
g=s . g s^{-1} \bmod G_{r+1}=\prod_{u} w\left(g_{u}^{\prime}, i, u\right) \prod_{u} w\left(g_{u}, i, u\right) \quad \bmod G_{r+1}=
$$




$$
=\prod_{u} w\left(g_{u}^{\prime} g_{u}, i, u\right) \quad \bmod G_{r+1} .
$$

Indeed, the elements $w\left(g_{u}, i, u\right)$ lie in $G_{r}$ and therefore they are central modulo $G_{r+1}$. For an arbitrary $u \in \cup_{i=1}^{n} M_{i}$, Hall identity $[x y, z]=$ $[x, z][[x, z], y][y, z]$ and multilineariy of $w$ imply that

$$
w\left(g_{u}^{\prime}, i, u\right) w\left(g_{u}, i, u\right)=w\left(g_{u}^{\prime} g_{u}, i, u\right) \quad \bmod G_{r+1} .
$$

That is, we have proved that if $G_{t} \subseteq S G_{r}$ for some $r \geq t$, then an arbitrary element $g \in G_{t}$ lies in $S G_{r+1}$, what proves our claim.

All $\mathbb{Z}_{p}$-modules $G_{i} / G_{i+1}$ are finitely generated and $p^{k}\left(G_{i} / G_{i+1}\right)=(0)$. This implies that each term $G_{i}$ of the lower central series has finite index in $G$. In particular, $\left|G: G_{t}\right|<\infty$.

Hence there exists a finite collection of elements $a_{1}, \ldots, a_{r} \in w(G)$ such that

$\langle\overline{w(G)}\rangle=\bigcup a_{i_{1}} \cdots a_{i_{\mu}} G_{t} \subseteq \bigcup a_{i_{1}} \cdots a_{i_{\mu}} S, 1 \leq \mu \leq\left|G: G_{t}\right| 1 \leq i_{1}, \ldots, i_{\mu} \leq r$.

Let $r^{\prime}=r\left|G: G_{t}\right|$ and let us repeat $\left|G: G_{t}\right|$ times the sequence $a_{1}, \ldots, a_{r}$

$$
a_{1}^{\prime}, \ldots, a_{r^{\prime}}^{\prime}=a_{1}, a_{2}, \ldots, a_{r}, a_{1}, a_{2}, \ldots, a_{r}, a_{1}, a_{2}, \ldots, a_{r} .
$$

Then

$$
\langle\overline{w(G)}\rangle=\bigcup a_{j_{1}}^{\prime} \cdots a_{j_{\mu}}^{\prime} S,
$$

where $1 \leq j_{1}<j_{2} \cdots<j_{\mu} \leq r^{\prime} ; 1 \leq \mu \leq\left|G: G_{t}\right|$.

Since each $a_{i}^{\prime} \in w(G)$, let $a_{i}^{\prime}=w\left(a_{i, 1}, \ldots a_{i, n}\right), 1 \leq i \leq r^{\prime}$ and $\alpha_{i}^{\prime}=$ $\left(a_{i, 2}, \ldots, a_{i, n}\right)$.

Then

$$
\langle\overline{w(G)}\rangle=w\left(G, 1, \alpha_{1}^{\prime}\right) \cdots w\left(G, 1, \alpha_{r}^{\prime}\right) S
$$

which implies that the word $w$ is strongly elliptic on $G$ and completes the proof of the lemma.

\section{Proof of Theorem 1.2}

Let $x_{1}, \ldots, x_{m}$ be generators of the group $\Gamma$. Then the Lie $\mathbb{Z}_{p^{-}}$algebra $L=L(G)$ is generated by elements $x_{i} G_{2}, 1 \leq i \leq m$. There exists $k \geq 1$ 
such that $x_{i}^{p^{k}}=1,1 \leq i \leq m$. This implies $p^{k} x_{i} G_{2}=0,1 \leq i \leq m$ and therefore $p^{k} L=(0)$. Hence $L$ can be viewed as a $\mathbb{Z} / p^{k} \mathbb{Z}$ - algebra.

The following result was proved in [MZ]. Let $\Gamma$ be a finitely generated residually- $p$ torsion group. Let $\Gamma=\Gamma_{1}>\Gamma_{2}>\cdots$ be its lower central series. Then an arbitrary homogenous element of the Lie ring $L(\Gamma)=\oplus_{i \geq 1} \Gamma_{i} / \Gamma_{i+1}$ is ad-nilpotent. This result implies that the Lie algebra $L=L(G)$ satisfies the assumptions of Lemma 3.1. Hence $|L: \operatorname{Spanf}(L)|<\infty$ and $f$ is strongly elliptic on $L$. Now, by Lemma 3.2 the word $w$ is strongly elliptic on the group $G$, which finishes the proof of Theorem 1.2.

\section{References}

[Go] E. S. Golod, On nil algebras and finitely approximable- p- groups, Izv. Akad. SSSR, Ser. Mat. 28, (1964), 273-276.

[Gr] R. Grigorchuk, Degrees of growth of finitely generated groups and the theory of invariant means. Izv. Akad. Nauk SSSR, Ser. Mat. 48 n. 5, (1984), 939-985.

[GuS] N. Gupta and S. Sidki, On the Burnside problem for periodic groups. Math. Z. 182 n. 3, (1983), 385-388.

$[\mathrm{H}] \mathrm{G}$. Higman, Lie rings methods in the theory of finite nilpotent groups, Cambridge Univ. Press, New York. 1958 Proc. Internat. Congress Math. (1960), 307-312.

[JZ] A. Jaikin-Zapirain, On the verbal witdth of finiteley generated prop-groups. Rev. Mat. Iberoam. 24, (2008), 617-630.

[Kl] B. Klopsch, Normal subgroups of Substitutions Groups of Formal Power Series, J. of Algebra 228, (2000), 91-106.

[K] A. I. Kostrikin, On local nilpotency of Lie rings that satisfy Engel's condition, Dokl. Akad. Nauk. SSSR 118, 1958 1074-1077.

[MZ] C. Martínez and E. Zelmanov, On Lie rings of torsion groups, Bull. Math. Sci 6, (2016), 371-377.

[P] B. J. Plotkin, Algebraic sets of elements in groups and Lie algebras, Uspehi Math. Nauk 13, no. 6, (1958), 133-138. 
[S] D. Seagal, Words: Notes on Verbal Width in Groups. London Math. Soc. Lecture Note Series 361, pp.121, 2009.

[Se] J. P. Serre, Cohomologie galoisienne. Springer Verlag 1964.

[Z1] E. Zelmanov, On the restricted Burnside problem. Proceedings of the International Congress of Mathematicians Kyoto 1990. Math. Soc. Japan, Tokyo 1991, 395-402.

[Z.2] E. Zelmanov, Nil rings and periodic groups. With a preface by Jongsik Kim. KMS Lecture Notes in Mathematics, Korean Math. Soc. Seoul 1992, x +79 pp.

[Z.3] E. Zelmanov, Lie algebras and torsion groups with identity, Submitted. Arxiv 160405678.

[ZSSS] K. A. Zhevlakov, A. M. Slinko, I. P. Shestakov and A. I. Shirshov, Rings that are nearly associative. Academic Press, ING. 1982. 